\title{
Autism spectrum disorders (ASD) in Africa: a perspective
}

\section{MO Bakare', KM Munir²}

${ }^{1}$ Child and Adolescent Unit, Federal Neuro-Psychiatric Hospital, New Haven, Enugu, Enugu State, Nigeria 2Developmental Medicine Center, Boston Children's Hospital, Boston, MA, USA

\begin{abstract}
Background: The universal occurrence of autism spectrum disorders (ASD) was queried about twenty-six years ago. It was thought to occur only in western industrialized countries with high technological development. Over the last decade, knowledge about ASD and its prevalence has been documented as being on the rise in different regions of the world, with most literature coming from the western world - the situation in Africa on aspects of ASD remain unclear. Methods: Literature cited in Pubmed over the last decade on aspects of epidemiology, diagnosis, aetiology and knowledge of ASD in the African context were assessed. Keywords: autism, diagnosis, aetiology, knowledge and Africa were variously combined in the literature search. Results: No study specifically addressed the epidemiology of ASD in Africa. One of the two studies that were relevant addressed epidemiology of ASD in Arab countries, though included two Northern African countries. A higher proportion of non-verbal cases of ASD compared to verbal cases was documented in literature coming from Africa. Associated co-morbid disorders included intellectual disability, epilepsy and oculo-cutaneous albinism. Aetiological factors postulated included post-encephalitic infection, genetic and auto-immune factors, and vitamin D deficiency. Knowledge about ASD in Africa was noted to be low. Conclusion: There is a need for epidemiological studies in Africa to define the magnitude of the problem of ASD and the characteristics of children affected by ASD in this region. This would help in planning and might be helpful in answering the question of aetiology of ASD. Policy making needs to be directed at issues of childhood developmental disorders in Africa.
\end{abstract}

Key Words: Epidemiology; Diagnosis; Aetiology; Knowledge; Autism; Africa

Received: 08-09-2010

Accepted: 07-10-2010

doi: http://dx.doi.org/10.4314/ajpsy.v14i3.3

\section{Introduction}

About two and half decades ago, the universality of autism spectrum disorders (ASD) was questioned. ${ }^{1}$ ASD was thought to occur mostly in Western cultures and countries with high technological development. ${ }^{1}$ Occurrence of ASD in Africa was then a subject of debate. Sanual ${ }^{1}$ concluded then that studies are required to address the open question of the universality of ASD. Over the last decade and a half, there has been documented evidence of an increase in the prevalence of ASD and knowledge about the disorder in other parts of the world. ${ }^{2}$ The present situation in Africa on various aspects of ASD is unclear. It is against this background that the present paper reviewed the literature cited in Pubmed related to various aspects of ASD over the last decade in Africa. Specifically aspects of epidemiology, diagnosis, aetiology and knowledge about ASD as documented in Pubmed cited literature published on ASD among Africans over the last decade was reviewed.

\section{Methods}

Keywords - autism, diagnosis, aetiology, knowledge and Africa were variously combined in a Pubmed search of the literature published on the topic of ASD in Africa for the period: January 2000 - December 2009. A total of twelve papers were published over the last decade on ASD among Africans. ${ }^{3-14}$ These papers addressed various aspects of ASD like epidemiology, diagnosis, aetiology and knowledge among Africans and in African countries. Four of the papers came from Nigeria, three from Egypt, two from Sweden, and one each from Tunisia and Tanzania. A further paper focused on Arab countries, but with the inclusion of Egypt and Tunisia among the countries where the study was conducted. Two of the twelve studies addressed aspects of epidemiology relating to the prevalence of ASD. Three studies addressed aspects of diagnosis relating to symptom presentation and associated co-morbidities. Six studies addressed aetiology of ASD among African children, while two studies addressed knowledge and awareness of ASD in Africa. Table I shows the country or region of origin of the papers and also various aspects of ASD addressed by this literature. 


\section{Results}

\section{Epidemiology}

Two studies addressed the aspect of prevalence of ASD among African children. Seif Eldin et $\mathrm{al}^{3}$ focused on Arab countries, but included two African countries (Egypt and Tunisia) in North Africa. The prevalence of ASD among children with developmental disorders in Egypt and Tunisia was documented as $33.6 \%$ and $11.5 \%$ respectively. ${ }^{3}$ These authors employed the Modified Checklist for Autism in Toddlers (M-CHAT) as a screening tool.

Barnevik-Olsson et $\mathrm{al}^{4}$ assessed the prevalence of ASD among children born to Somali parents living in Sweden. They documented a significantly higher prevalence of ASD among children of Somali parents living in Sweden compared to the non-Somali group. Children of Somali parents living in Sweden were found to have a prevalence of $0.7 \%$ ASD compared with approximately $0.2 \%$ in the non-Somali group. Barnevik-Olsson et al ${ }^{4}$ concluded that their findings warrant further investigation into why children of Somali parents living in Sweden tend to have a higher prevalence of ASD, suggesting that Vitamin D deficiency (among other factors) might be responsible for this finding

\section{Diagnosis}

Three studies ${ }^{5-7}$ provided information on diagnosis of ASD among African children, as it relates to symptom presentation and co-morbid disorders.

\section{Symptoms at presentation}

Aside from the core symptoms of ASD in the areas of impairment in social interaction, communication and restricted, repetitive repertoire of behavior, one common symptom presentation that was found in over fifty percent of the cases reported in the literature was lack of expressive language (non-verbal cases). Belhadj et $\mathrm{al}^{5}$ reported $51.2 \%$ of non-verbal cases of ASD in their study. Mankoski et al ${ }^{6}$ observed about $71 \%$ of non-verbal cases of ASD in their study, but they quickly concluded that this observation might be as a result of local diagnostic practices. The inference of these findings is that based on current diagnostic practice, the proportion of non-verbal cases diagnosed with ASD in Africa might be greater than the proportion of cases who are verbal.

\section{Co-morbid disorders}

Among the co-morbid disorders diagnosed in association with ASD among African children, intellectual disability was more common. Belhadj et $\mathrm{al}^{5}$ documented co-morbid intellectual disability in over 60\% of cases studied. Other documented comorbid disorders in association with ASD among African children included epilepsy ${ }^{5}$ and, in one case, oculo-cutaneous albinism. ${ }^{\text {? }}$

\section{Aetiology}

Six papers addressed aetiological factors for ASD among African children. Five of these based the proposed etiological factors on scientific methodologies ${ }^{6-11}$, while one assessed the opinion of healthcare workers about the aetiology of ASD. ${ }^{12}$ Common aetiological factors proposed for ASD among African children included post-encephalitic infection or sepsis preceding onset of symptoms of $\mathrm{ASD}^{6}$, genetic and auto-immune factors ${ }^{8,10,11}$ and vitamin $D$ deficiency. ${ }^{9}$ The only study that assessed the opinion of healthcare workers on aetiology of ASD documented a very significant proportion ( $43 \%$ ) of the healthcare workers subscribing to preternatural and supernatural aetiological factors for $\mathrm{ASD}^{12}$, a finding further substantiating the inseparable spiritual beliefs of Africans and aetiological explanations of neuropsychiatric disorders.

\section{Knowledge about ASD}

Two papers addressed the extent of knowledge about ASD. The findings of these two studies inferred that knowledge and awareness about ASD is still low in Africa, especially sub-Saharan Africa. 13,14 They concluded that continuous education of healthcare workers and the general public in Africa is essential to raise the level of knowledge and awareness about ASD, as improved knowledge would help in early recognition and intervention for African children with ASD.

\section{Discussion}

The debate raised about two and half decades ago that questioned the occurrence of ASD in Africal has been put to rest to a large extent by research addressing aspects of ASD in Africa over the last decade. Based on the present state of knowledge, there is no element of doubt that ASD does occur among African children living both in Africa and abroad. However, many questions still remain unanswered about the definitive prevalence, aetiology and characteristics of children affected by ASD in Africa, especially sub-Saharan Africa.

The two studies ${ }^{3,4}$ that addressed the aspect of epidemiology of ASD among Africans did not assess the prevalence of ASD in the context of the population of children living in Africa. Seif Eldin et $\mathrm{al}^{3}$ specifically studied Arab countries and Barnevik-Olsson et $\mathrm{al}^{4}$ determined prevalence of ASD among Somali immigrants living in Sweden.

The significant proportion of cases of ASD who lack expressive speech (non-verbal cases) ${ }^{5,6}$ needs to be verified in a large epidemiological study beyond the clinic-based approach to date. Co-morbidity of epilepsy and intellectual disability in association with ASD reported from Tunisia is an interesting finding ${ }^{5}$ as it corroborates the observation of Mankoski et $\mathrm{al}^{6}$ that ASD in Africa is rarely diagnosed exclusive of intellectual disability. The possibility also exists that the more severe cases of

\section{Table I: Country or region of origin and various aspects of ASD addressed by the papers included in the study}

\begin{tabular}{|l|l|l|l|}
\hline Country or Region of Origin & Number of Studies & Aspects of ASD & Number of Studies \\
\hline Nigeria & 4 & Epidemiology & 2 \\
Egypt & 3 & Diagnosis & 3 \\
Sweden & 2 & Aetiology & 6 \\
Tunisia & 1 & Knowledge & 2 \\
Tanzania & 1 & & \\
Arab populations (Tunisia \& Egypt) & 1 & & \\
\hline
\end{tabular}


ASD are referred to orthodox medical practice for help, as the majority of cases that are less severe are likely to seek help from spiritualists and traditional healers. This stems from the perceived etiological explanation of ASD among African healthcare workers, as documented by a recent study.12 Therefore, the relationship between ASD and intellectual disability among African children requires further exploration.

The proportion of children who present with symptoms of ASD post-encephalitic infection or sepsis (as documented by Mankoski et al ${ }^{6}$ ) might be substantially higher among African children when compared to what appears to be the case in most developed countries. This may translate cumulatively to a higher prevalence of ASD among African children compared to children from the western world. The hypothesis that pre-natal Vitamin D deficiency may contribute to aetiology of ASD as proposed by BarnevikOlsson et $\mathrm{al}^{4}$ in the Swedish immigrant study would need further confirmation through study of ASD in the tropical and sub-tropical regions of Africa, where Vitamin D deficiency is likely to be rare because of the availability of abundant ultra violet-B radiation from sunlight. On the other hand, the general population prevalence of ASD among African children may be lower than found in Western societies because of certain environmental factors including the proposed Vitamin D hypothesis. ${ }^{4}$ The proposition of Sanua ${ }^{1}$, that ASD is a disease of western civilization and more prevalent in highly industrialized countries with high technological development, may still hold true. Epidemiological data on ASD in sub-Saharan African children would not only clarify the present status of ASD in this region, but it would also help in further understanding the possible aetiology of ASD.

The low level of knowledge and awareness about ASD, as documented by recent studies from Africa ${ }^{13,14}$, is a call for engaging in activities that can improve the level of knowledge and awareness about ASD among healthcare workers and the general population in Africa. This would help early recognition of cases and interventions which have been shown to improve prognosis. ${ }^{15,16}$ It would also help in changing popular perceptions in Africa regarding a spiritual explanation for ASD. ${ }^{12}$

\section{Child and adolescent mental health services, human resources and policy making in Africa}

It is important to pay attention to the recurrent theme of scarce child and adolescent mental health legislation, policies, services, programmes and human resources in Africa. ${ }^{17,18}$ The need to scale up services to meet the challenges of child and adolescent mental health in Africa through mass education, personnel training, and appropriate policy formulation is imperative. The modalities stated by Patel et $\mathrm{al}^{17}$ in meeting the challenges of scarce resources in the area of child and adolescent mental health in Africa and other low income countries is worth giving thoughtful consideration.

\section{Conclusion}

Studies are required, specifically epidemiological studies, to define the magnitude of the problem of ASD as well as the characteristics of children with ASD in Africa, especially subSaharan Africa. These might also help answer the bothering question regarding the aetiology of ASD and may also shed light on the reasons for possible differences in prevalence between geographical regions, if any exist. Africa also needs more policy making attention directed at child and adolescent mental health service provision, especially regarding the issues of childhood developmental disorders and intellectual disability.

\section{References}

1. Sanua VD. Is infantile autism a universal phenomenon? An open question. Int J Soc Psychiatry 1984; 30(3): 163 - 177.

2. Fombonne E. Epidemiological surveys of autism and other pervasive developmental disorders: an update. J Autism Dev Disord 2003: 33: 365 - 382.

3. Seif Eldin A, Habib D, Noufal A, Farrag S, Bazaid K, Al-Sharbati M, et al. Use of M-CHAT for a multinational screening of young children with autism in the Arab countries. Int Rev Psychiatry 2008; 20(3): $281-289$.

4. Barnevick-Olsson, M Gillberg C, Fernell E. Prevalence of autism in children born to Somali parents living in Sweden: a brief report. Dev Med Child Neurol 2008; 50(8): 598 - 601.

5. Belhadj A, Mrad R, Halayem MB. A clinic and paraclinic study of Tunisian population of children with autism. About 63 cases. Tunis Med 2006; 84(12): 763 - 767.

6. Mankoski RE, Collins M, Ndosi NK, Mgalla EH, Sarwatt VV, Folstein SE. Etiologies of autism in a case-series from Tanzania. J Autism Dev Disord 200; 36(8): 1039 - 1051.

7. Bakare MO, Ikegwuonu NN. Childhood autism in a 13 year old boy with oculo-cutaneous albinism: a case report. J Med Case Reports 2008; $2: 56$.

8. Mostafa GA, El-Sayed ZA, El-Aziz MM, El-Sayed MF. Serum antimyelin-associated glycoprotein antibodies in Egyptian autistic children. J Child Neurol 2008; 23(12): 1413 - 1418.

9. Bejerot S, Humble, M. Increased occurrence of autism among Somali children- does vitamin D deficiency play a role? Tidsskr Nor Laegeforen. 2008; 128(17): 1986 - 1987.

10. Mostafa GA, El-Hadidi ES, Hewedi DH, Abdou MM. Oxidative stress in Egyptian children with autism; relation to autoimmunity. $J$ Neuroimmunol 2010; 219(1-2): 114-118.

11. Mostafa GA, Kitchener N. Serum anti-nuclear antibodies as a marker of autoimmunity in Egyptian autistic children. Pediatr Neurol 2009; 40(2): 107 - 112.

12. Bakare MO, Agomoh AO, Ebigbo PO, Eaton J, Okonkwo KO, Onwukwe JU, Onyeama GM. Etiological explanation, treatability and preventability of childhood autism: A survey of Nigerian healthcare workers' opinions. Annals of General Psychiatry 2009; 8: 6.

13. Bakare MO, Ebigbo PO, Agomoh AO, Menkiti NC. Knowledge about childhood autism among health workers (KCAHW) questionnaire: description, reliability and internal consistency. Clinical Practice and Epidemiology in Mental Health 2008; 4:17.

14. Bakare MO, Ebigbo PO, Agomoh AO, Eaton J, Onwukwe JU, Onyeama GM, et al. Knowledge about childhood autism and opinion among healthcare workers on availability of facilities and law caring for the needs and rights of children with childhood autism and other developmental disorders in Nigeria. BMC Pediatrics 2009; 9: 12.

15. Gray KM. Are there early features of autism in infants and preschool children? J Paediatr Child Health 2001; 37: 221 - 226.

16. American Academy of Pediatrics, Committee on Children with Disabilities. The pediatrician's role in the diagnosis and management of autistic spectrum disorders in children. Pediatrics 2001; 107: 1221 - 1226.

17. Patel V, Flisher AJ, Nikapota A, Malhotra S. Promoting child and adolescent mental health in low and middle income countries. J Child Psychol Psychiatry 2008; 49(3): 313 - 334.

18. Kleintjes S, Lund C, Flisher AJ. MHAPP Research Programme Consortium. A situation analysis of child and adolescent mental health services in Ghana, Uganda, South Africa and Zambia. Afr J Psychiatry 2010; 13(2): $132-139$. 